\title{
The T2K experiment and its time projection chambers
}

\section{HANSEN ${ }^{* \dagger}$}

IFIC (CSIC, Univ. Valencia)

E-mail: christian.hansen@ific.uv.es

\section{MONFREGOLA ${ }^{* \dagger}$}

IFIC (CSIC, Univ. Valencia)

E-mail: laura.monfregola@ific.uv.es

\section{RAVONEL}

University of Geneva

E-mail: melody.ravonel@cern.ch

The Tokai to Kamioka (T2K) experiment [1] is a long baseline neutrino oscillation experiment located in Japan and its goal is to gain a more complete understanding of the neutrino oscillation parameters. A highly pure muon neutrino beam is directed from the accelerator center JPARC towards the Super-Kamiokande (SK) detector, which is $295 \mathrm{~km}$ away. A key element of the design of the T2K facility is the use of an off-axis technique. The main physics goals of T2K are to measure the mixing angle $\theta_{13}$ in a $v_{e}$ appearance experiment and improve the measurement of the atmospheric parameters $\Delta m_{23}^{2}$ and $\theta_{23}$ using the $v_{\mu}$ disappearance channel. The near detector (ND280), located $280 \mathrm{~m}$ away from the target, will measure the neutrino beam properties and the neutrino interaction cross section and kinematics before the oscillation, in order to predict the relevant neutrino interactions at SK. It consistes of a magnet, a Pi-Zero Detector (optimized for measuring the rate of neutral current $\pi_{0}$ production), an electromagnetic calorimeter (whose main purpose is to measure the photons produced in ND280), a Side Muon Range Detector (to measure the range of muons that exit the sides of ND280) and a tracker (optimized for measuring the momenta of charged particles). The tracking device consists of a sandwich of three time projection chambers (TPCs) and two fine grained detectors (FGDs) which provide the target material. An overview of the TPC calibration methods and some results from the tests done at CERN to study the performance of the TPC readout modules are presented.

10th International Workshop on Neutrino Factories, Super beams and Beta beams

June 30 - July 52008

Valencia, Spain

\footnotetext{
* Speaker.

† On behalf of the T2K TPC group (IFIC Valencia, Geneva, IFAE Barcelona, TRIUMF, British Columbia, Victoria, DSM/Dapnia, CEA/Saclay, RWTH Aachen, LPNHE Paris)
} 


\section{T2K-ND280-TPCs}

The ND280-TPCs will measure the momentum of muons from CCQE events $\left(v_{\mu}+n \rightarrow\right.$ $\mu^{-}+p$ ) to provide an accurate neutrino energy spectrum. $\mathrm{dE} / \mathrm{dx}$ measurements in the TPCs will be used to identify the $v_{e}$ component in the beam that is one of the major backgrounds to the $v_{e}$ appearance measurement in SK. The MicroMegas (MM) technology [2,3] is used for the readout of the TPCs to achieve the design criteria of compactness and high segmentation, necessary to obtain the required momentum resolution (10\% at $1 \mathrm{GeV} / \mathrm{c})$. A MM module consists of a grounded pad plane with a micro-mesh $128 \mu \mathrm{m}$ above. 12 pillars over each pad are supporting the mesh to maintain a constant gap. The drift field inside this gap is about $30000 \mathrm{~V} / \mathrm{cm}$ and allows the multiplication of the primary electrons. The avalanche is then collected by the pads. $72 \mathrm{MM}$ modules, each $36 \times 34 \mathrm{~cm}$, are needed to instrument the 3 TPCs (12 modules for each of the 2 readout planes of each TPC). The MM modules are produced by Saclay and testing of the modules is done at CERN by all European insitutes participating in the TPC.

\section{Cosmic Ray Test at CERN}

A MM module was inserted into the HARP TPC at CERN to study the response to real particles (cosmics) in a magnetic field [3]. In particular measurements at the same magnetic field as in ND280 (0.2 Tesla) have been taken. This test demonstrated a spatial resolution of about $0.6 \mathrm{~mm}$ (see Fig. 1), which meets the design specifications.

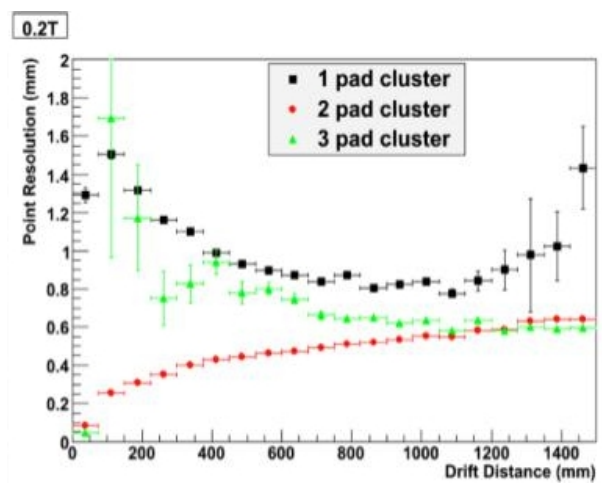

Figure 1: Point resolution as a function of the drift distance for one, two and three pad clusters in a $0.2 \mathrm{~T}$ magnetic field.

\section{TPC Calibration}

To deal with unforeseen distortions, due to e.g. electric and magnetic field inhomogeneities, attenuation, gain variation, MM module displacement, cross talk, etc., and obtain the required resolution, an elaborated calibration scheme is being established. In particular measured field maps, a laser system (see sec. 3.1) and cosmics rays will be used to correct spatial distortions due to the imperfections in the electric and magnetic fields, mechanics and gas properties; the drift velocity will be measured using the laser system, cosmic rays and an external monitor chamber, while the 

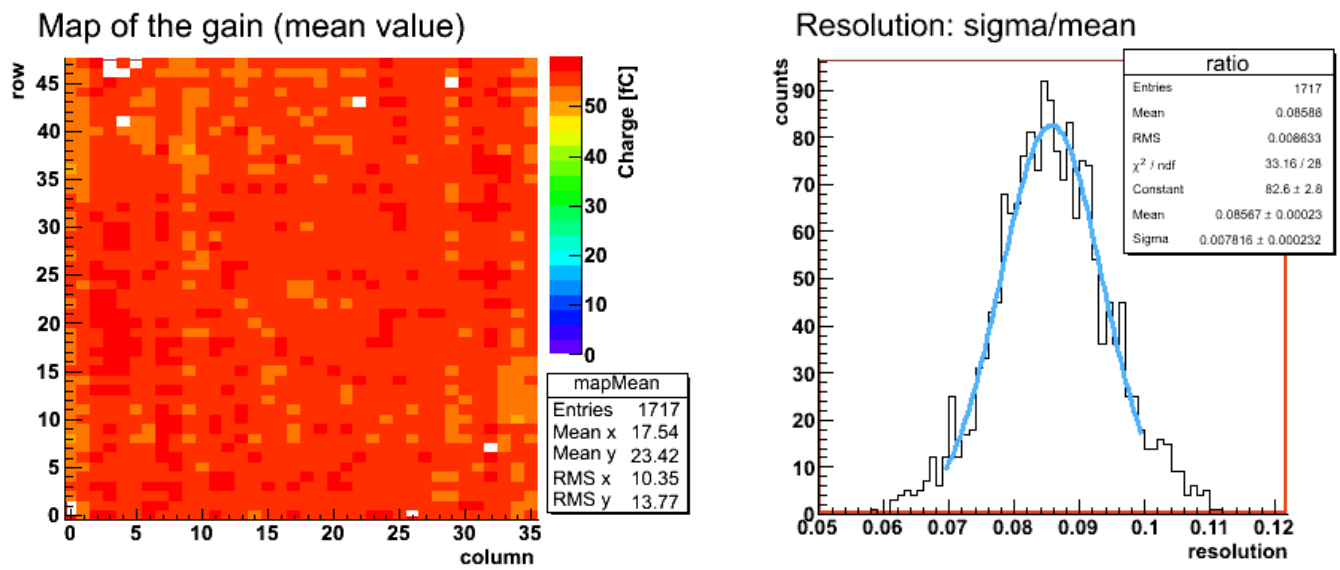

Figure 2: MM's gain map (left) and energy resolution distribution (right) provided by the Test Bench. Each MM module contains 1728 pads arranged in 36 columns and 48 rows (pad size: $9.7 \times 6.9 \mathrm{~mm}$ ).

test bench (see sec. 3.2) will be used to study the gain of the MM modules. Some of the calibration methods are offline, like the test bench, and some will be online, like the laser calibration system.

\subsection{Laser Calibration System}

The TPC is foreseen to have a laser calibration system. The idea is that UV light will be transported from the laser by optical fibers and then spread by lenses on to the central cathode where a collection of aluminium dots with a well defined pattern will emit photoelectrons to be drifted. The pattern will produce an image on the pad plane that will be detected and used for the calibration. This method will be used to correct spatial distortions and for a continuous monitoring of the drift velocity.

\subsection{Test Bench}

The test bench [4] consists of a drift chamber with a MM module implemented on one side and a cathode on the other side. The chamber is held vertically and mounted on a support structure in front of x-y stages. The x-y stages are used to move a strong ${ }^{55} \mathrm{Fe}$ source behind the cathode over each pad during the calibration. Two other weak sources of iron are fixed behind the cathode for a stability control in time. In order to maximize the gain, the calibration box is filled with $\mathrm{ArCF}_{4} \mathrm{iC}_{4} \mathrm{H}_{10}$ (95:3:2). The goal of the test bench is to provide a pad-per-pad characterization for a proper track and energy reconstruction. In particular it provides a map of gain and energy resolution for each MM module. The gain variation within a module is demonstrated to be about $2.3 \%$ (Fig. 2-left) while the energy resolution is of the order of $8.6 \%$ (Fig. 2-right).

\section{References}

[1] Y. Itow et al., [arXiv:hep-ex/0106019v1]

[2] Y. Giomataris et al., Nucl. Instr. and Meth. A 376 (1996) 29

[3] J. Bouchez et al., Nucl. Instr. and Meth. A 574 (2007) 425-432

[4] M.Di Marco for the T2K TPC groups, J. Phys.: Conf. Ser. 65012019 